\title{
Clinical-dosimetric analysis of measures of dysphagia including gastrostomy-tube dependence among head and neck cancer patients treated definitively by intensity-modulated radiotherapy with concurrent chemotherapy
}

\author{
Baoqing Li ${ }^{1}$, Dan $\mathrm{Li}^{2}$, Derick H Lau ${ }^{3}$, D Gregory Farwell ${ }^{4}$, Quang Luu ${ }^{4}$, \\ David M Rocke ${ }^{2,5}$, Kathleen Newman ${ }^{1}$, Jean Courquin ${ }^{1}$, James A Purdy ${ }^{1}$ and \\ Allen M Chen*1
}

\begin{abstract}
Address: ${ }^{1}$ Departments of Radiation Oncology, University of California Davis Cancer Center, Sacramento, CA 95817, USA, ${ }^{2}$ Departments of Applied Science, University of California Davis Cancer Center, Sacramento, CA 95817, USA, ${ }^{3}$ Departments of Medical Oncology, University of California Davis Cancer Center, Sacramento, CA 95817, USA, ${ }^{4}$ Departments of Otolaryngology-Head and Neck Surgery, University of California Davis Cancer Center, Sacramento, CA 95817, USA and ${ }^{5}$ Departments of Public Health Sciences, University of California Davis Cancer Center, Sacramento, CA 95817, USA

Email: Baoqing Li - bao-qing.li@ucdmc.ucdavis.edu; Dan Li - danli@ucdavis.edu; Derick H Lau - derick.lau@ucdmc.ucdavis.edu; D Gregory Farwell - gregory.farwell@ucdmc.ucdavis.edu; Quang Luu - quang.lu@ucdmc.ucdavis.edu;

David M Rocke - david.rocke@ucdmc.ucdavis.edu; Kathleen Newman - kathleen.newman@ucdmc.ucdavis.edu;

Jean Courquin - jean.courquin@ucdmc.ucdavis.edu; James A Purdy - james.purdy@ucdmc.ucdavis.edu;

Allen M Chen* - allen.chen@ucdmc.ucdavis.edu

* Corresponding author
\end{abstract}

Published: 12 November 2009

Radiation Oncology 2009, 4:52 doi:10.1 186/1748-717X-4-52

This article is available from: http://www.ro-journal.com/content/4/I/52

(C) 2009 Li et al; licensee BioMed Central Ltd.

This is an Open Access article distributed under the terms of the Creative Commons Attribution License (http://creativecommons.org/licenses/by/2.0), which permits unrestricted use, distribution, and reproduction in any medium, provided the original work is properly cited.
Received: 15 June 2009

Accepted: 12 November 2009

\begin{abstract}
Purpose: To investigate the association between dose to various anatomical structures and dysphagia among patients with head and neck cancer treated by definitive intensity-modulated radiotherapy (IMRT) and concurrent chemotherapy.

Methods and materials: Thirty-nine patients with squamous cancer of the head and neck were treated by definitive concurrent chemotherapy and IMRT to a median dose of 70 Gy (range, 68 to 72). In each patient, a gastrostomy tube (GT) was prophylacticly placed prior to starting treatment. Prolonged GT dependence was defined as exceeding the median GT duration of 192 days. Dysphagia was scored using standardized quality-of-life instruments. Dose-volume histogram (DVH) data incorporating the superior/middle pharyngeal constrictors (SMPC), inferior pharyngeal constrictor (IPC), cricoid pharyngeal inlet (CPI), and cervical esophagus (CE) were analyzed in relation to prolonged GT dependence, dysphagia, and weight loss.

Results: At 3 months and 6 months after treatment, $87 \%$ and $44 \%$ of patients, respectively, were GT dependent. Spearman's $\rho$ analysis identified statistical correlations $(p<0.05)$ between prolonged GT dependence or high grade dysphagia with IPC V65, IPC V60, IPC Dmean, and CPI Dmax. Logistic regression model showed that IPC V65 > 30\%, IPC V $60>60 \%$, IPC Dmean $>60$ Gy, and CPI Dmax > 62 Gy predicted for greater than $50 \%$ probability of prolonged GT dependence.
\end{abstract}


Conclusion: Our analysis suggests that adhering to the following parameters may decrease the risk of prolonged GT dependence and dysphagia: IPC V65 < I5\%, IPC V60 < 40\%, IPC Dmean $<55$ Gy, and CPI Dmax < 60 Gy.

\section{Introduction}

Concurrent chemoradiation therapy using intensity-modulated radiotherapy (IMRT) has gained widespread acceptance as a definitive treatment for locally advanced head and neck cancer due to significant improvement in tumor control and organ preservation with the addition of chemotherapy, and promising advantage of increasing therapeutic gain using IMRT technique [1-4]. However, it is becoming increasingly clear that chemoradiation strategy is associated with an increased incidence and severity of swallowing-related toxicities, including high-grade dysphagia, severe weight loss, and prolonged dependence on gastrostomy tube (GT) for fluid and nutritional support [5-7].

Indwelling GT has been shown to compromise quality of life because it may cause infection and physical discomfort, distort patient's self-esteem, and induce anxiety, depression, and social isolation [8]. Presently there is a lack of data associating GT dependence and dosimetric parameters among patients undergoing definitive chemoradiotherapy using IMRT for head and neck cancer $[9,10]$. This is of practical significance since, as a result of IMRT optimization, radiation doses can potentially be "dumped" to unspecified anatomical areas including those related to dysphagia that have not yet been rigorously investigated [11]. In a prospective trial using IMRT, Feng et al demonstrated the importance of monitoring dose to the pharyngeal constrictor muscles, the cervical esophagus (CE), and the glottic and supraglottic larynx (GSL) [12]. The purpose of the present study was to investigate the potential association between radiation dose to these structures vital for swallowing and severity of dysphagia, notably prolonged GT dependence, among a cohort of patients undergoing definitive IMRT chemoradiation for locally advanced head and neck cancer.

\section{Methods and materials \\ Patient characteristics}

This was a retrospective study approved by the Institutional Review Board at the University of California, Davis (UCD). Between January 2003 and January 2007, fortyeight patients with newly diagnosed squamous cell carcinoma involving the oral cavity, oropharynx, larynx or hypopharynx were treated with definitive chemoradiation consisting of IMRT and cisplatin at the UCD Cancer Center. Seven patients who either developed locoregional recurrence or were lost during follow up were excluded from the study. Two patients who refused prophylactic placement of a GT were also excluded. The remaining 39 patients included in the study. The median follow up was 15.6 months (range, 4.5 to 52 months), with 27 patients followed greater than 1 year. All patients received prophylactic placement of a GT prior to starting treatment. The GT was subsequently removed upon resolution of high grade dysphagia and stabilization of weight after treatment. Physician judgment if GT needed to be maintained was based on the criteria that 1) the patient's weight could not be maintained with less than two cans of supplemental feeding per day, or 2) the patient could not tolerate solid food without complaints of dysphagia,

Table I: Patient and tumor characteristics.

\begin{tabular}{|c|c|c|}
\hline Variable & No. patients & $\%$ \\
\hline Age & Mean 56, & \\
\hline Continuous & range $32-77$ & \\
\hline \multicolumn{3}{|l|}{ Gender } \\
\hline Male & 32 & 82 \\
\hline Female & 7 & 18 \\
\hline \multicolumn{3}{|l|}{ Active smoking* } \\
\hline Yes & 30 & 77 \\
\hline No & 9 & 13 \\
\hline \multicolumn{3}{|l|}{ Alcohol use } \\
\hline Heavy** & 11 & 28 \\
\hline Others & 28 & 72 \\
\hline \multicolumn{3}{|l|}{ KPS } \\
\hline $80-100$ & 25 & 64 \\
\hline $60-70$ & 14 & 36 \\
\hline \multicolumn{3}{|l|}{ Primary site } \\
\hline Oral cavity & 2 & 5 \\
\hline Oropharynx & 25 & 64 \\
\hline Larynx & 6 & 15 \\
\hline Hypopharynx & 3 & 8 \\
\hline Unknown primary & 3 & 8 \\
\hline \multicolumn{3}{|l|}{$T$ stage } \\
\hline TO, I, 2 & 25 & 64 \\
\hline T3, 4 & 14 & 36 \\
\hline \multicolumn{3}{|l|}{$N$ stage } \\
\hline No/NI & 16 & 41 \\
\hline N2 & 19 & 49 \\
\hline N3 & 4 & 10 \\
\hline \multicolumn{3}{|l|}{ Chemo regimen } \\
\hline CDDP-based & 33 & 85 \\
\hline Others & 6 & 15 \\
\hline \multicolumn{3}{|c|}{ Post $R T$ neck dissection } \\
\hline Yes & 5 & $13 \%$ \\
\hline No & 34 & $87 \%$ \\
\hline
\end{tabular}

*: currently smoking or smoking history within one year.

**: self reported active heavy alcohol drinking or more than one 6pack of beers per day. 
odynophagia or aspiration. None of the patients required GT reinsertion once the GT was initially removed after completion of radiation therapy. Table 1 shows patient characteristics of the study population.

\section{Target volume delineation}

The gross tumor volume (GTV) was specified as the gross extent of tumor as demonstrated by preoperative imaging and physical examination including endoscopy. Grossly positive lymph nodes were defined as any lymph nodes greater than $1 \mathrm{~cm}$ or those with a necrotic center. The high-risk clinical target volume (CTV1) was defined as the GTV plus a margin of $1-2 \mathrm{~cm}$ to account for microscopic disease spread. The CTV2 generally included the prophylactically treated cervical and supraclavicular neck. A CTV3 was also created to designate an area at lowest risk within the prophylactically treated low neck. The low neck was encompassed within the IMRT plan in all cases, and thus a separate anterior low-neck field was not used. Depending on disease site, the planning target volume (PTV) contained an automated $0.5 \mathrm{~cm}$ expansion of the CTV surfaces to account for patient setup error to create PTV1, PTV2, and PTV3, if necessary. The tumor volumes and sensitive normal structures were delineated on serial treatment planning CT images. Structures considered to be critically at risk included the spinal cord, optic nerves, optic chiasm, orbits, lens, brainstem, and parotid glands. No overlap between CTVs and uninvolved critical adjacent tissues was permitted for optimization purposes.

\section{Dose specification}

For patients receiving definitive radiation therapy, treatment plans were designed to provide a dose of 68 to $72 \mathrm{~Gy}$ (median, $70 \mathrm{~Gy}$ ) to $95 \%$ or greater of the PTV1 while spar-
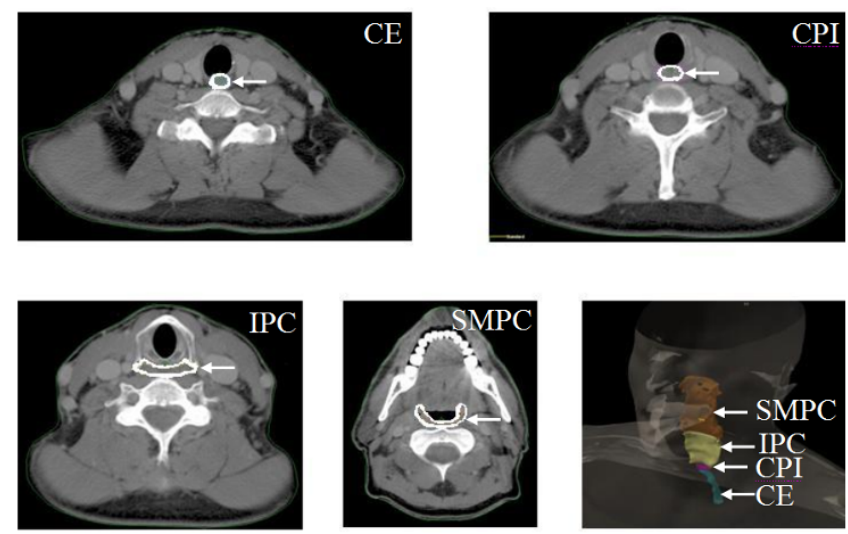

Figure I

Delineation of the swallowing structures on axial slices from simulation $C T$ and $3 D$ reconstructed image. SMPC = superior and middle pharyngeal constrictor; IPC = inferior pharyngeal constrictors; CPI = cricoid pharyngeal inlet; and CE = cervical esophagus. ing neighboring critical structures. The prescribed dose to PTV3 was 54 to 56 Gy. Dose to PTV2 ranged from 59.4 to 63 Gy (median, 60 Gy). For critical normal structures, dose constraints were designed to limit the maximum dose, whenever possible, to $1 \%$ of the volume to $54 \mathrm{~Gy}$ for the brainstem and optic nerves, 45 Gy for the spinal cord and optic chiasm, 60 Gy for the temporal lobes, and $30 \mathrm{~Gy}$ to $50 \%$ of the contralateral parotid gland. Treatment was by continuous-course IMRT with once-a-day treatment. Because our goal was to prescribe $1.8 \mathrm{~Gy}$ per fraction to the PTV2 daily, the PTV1 received a higher dose per fraction, typically 2.0 Gy or 2.12 Gy per fraction, and PTV3 typically 1.6-1.7 Gy per fraction.

\section{Delineation of swallowing structures}

The IMRT treatment plans of all 39 patients treated by definitive chemoradiation were retrieved from archival records. With the help of a board-certified head and neck surgeon, the swallowing structures were contoured on axial CT slides as previously described [11-14] (Fig. 1). Briefly, the pharyngeal constrictor (PC) was outlined as a single structure for which the cranial-most extent was the caudal tips of the pterygoid plates and the caudal-most extent was the inferior border of the cricoid cartilage. For purposes of analysis, the constrictors were considered as one structure and were also schematically divided into two parts: the superior and middle PC (SMPC) was defined from the caudal tips of the pterygoid plates through the lower edge of the hyoid, at the level of C2, C3 and upper C4. The inferior PC (IPC) was defined from below the hyoid through the inferior edge of the cricoid, with attachment to the inferior horn of thyroid cartilage, at the level of lower C4, C5 and upper C6. On non-contrast CT images, IPC can be identified as a structure with faint enhancement of mucosa surrounded by a thin intramural fat plate which facilitates the exclusion of the posterior cricoarytenoid muscle. The cricopharyngeal inlet (CPI) was defined as an oval structure of $1 \mathrm{~cm}$ in length, with lack of intramural fat plate. It extends from the caudal cricoid to the first tracheal ring, and is located at the level of lower C6. The CE was contoured as a round structure, caudal to the CPI, with its caudal-most extent corresponding to the thoracic inlet. With the above structures delineated on the axial CT slices, tabular differential dosevolume histogram (DVH) data for all the structures were re-computed, taking into consideration the dose actually delivered.

\section{Chemotherapy regimens}

The majority $(85 \%)$ of the patients received bolus cisplatin $\left(100 \mathrm{mg} / \mathrm{m}^{2}\right)$ given every 3 weeks on days 1 and 22 . The remaining patients received either weekly carboplatin $(\mathrm{AUC}=2)$ or weekly paclitaxel $\left(50 \mathrm{mg} / \mathrm{m}^{2}\right)$ for 6 weeks. Cetuximab was not used among any of the patients in the 
study. None of the patients received sequential induction or consolidation therapy.

\section{GT management}

The GT was inserted by the Department of Interventional Radiology at UCD, and was changed every three months. In 3 patients ( $8 \%$ ), additional GT changes were performed due to complications such as infection or obstruction. Patients were encouraged to undergo feeding by mouth for as long as it was tolerable. Body weight and toxicity (dysphagia, xerostomia, mucositis, nausea, vomiting, constipation, diarrhea, dysguesia, difficulties chewing) were assessed and addressed with patients weekly. We used the American Dietetic Association Medical Nutrition Therapy (MNT) Protocol for Cancer (Radiation Oncology) and the UCD Enteral Nutrition Guidelines. Decisions to wean off enteral feeds were based on individual patient and chemoradiation-induced toxicities with specific emphasis placed on inability to consume adequate oral nutrition and fluid, dysphagia, and prevent uncontrolled involuntary weight loss. Patients were weaned off enteral nutrition support when 1) the patient's weight could be maintained with less than two cans of supplemental feed per day, and 2) the patient could have certain solid food without complaints of dysphagia, odynophagia or aspiration.

\section{Follow-up evaluation}

Patients were typically seen 2 to 3 weeks after completion of radiation therapy and then every 3 months thereafter for the first year, every 6 months for the second and third year, and then annually. The mean follow up time was 16.2 months (range 4.5-52 months). If a persistent neck node was found on physical examination after completion of IMRT and/or was positive on PET/CT at 2 months follow-up, salvage neck dissection was performed. Duration of GT was defined as the interval between RT completion and the date of its removal, or until the date of last follow-up or death if the GT was still present. Prolonged GT dependence was defined as GT more than the median GT duration. Two patients whose follow-up time were less than the mean GT duration were excluded from further analysis of clinical-dosimetric association. Body weight and patient-reported dysphagia were recorded during each follow up. The lowest body weight during the follow up period was used. Severe weight loss was defined as more than 15\% weight loss. Patient-reported dysphagia was assessed with the validated UWQOL questionnaires given to patients during each follow-up visit. It contained one swallowing question with five possible answers ("I swallow normally", (grade 0); "I cannot swallow certain solid food", (grade 1); "I can only swallow soft food", (grade 2); "I can only swallow liquid food", (grade 3); and "I cannot swallow", (grade 4). Grade 3 and grade 4 defined high grade dysphagia. Mucositis and xerostomia was evaluated weekly during treatment, and at follow-up, based on Common Terminology Criteria for Advanced Events (CTCAE), version 2.0. Accordingly, high grade mucositis was defined as confluent pseudomembranous reaction with continuous patches $>1.5 \mathrm{~cm}$ (grade 3 ) or necrosis or deep ulceration; this may include bleeding not induced by minor trauma or abrasion (grade 4 ).

\section{Transnasal esophagoscopy (TNE), flexible endoscopic evaluation of swallow (FEES), and aspiration pneumonia work up}

Patients with grade 2 or greater dysphagia beyond 3 months after radiation were referred for TNE. The TNE technique has previously been described $[15,16]$. At the discretion of the physician, a FEES or aspiration pneumonia work up (bacterial culture and chest X-ray) was performed. FEES allows direct visual assessment of many swallowing functions including muscular function, premature spillage, pooling, laryngeal penetration, and presence of aspiration. In brief, the patients were examined seated upright without anesthesia. Liquid (colored water), pureed food (yogurt), and chewable food (bread) were ingested while the hypopharynx and laryngeal contents were viewed with the fiberscope. The results were scored as "little", "moderate", or "severe" using the following variables: residue, penetration, and aspiration of three different types of diet (water, yogurt, and bread), and mucus stases. Aspiration pneumonia was defined as cultured bacterial pneumonia with radiographic evidence of infiltration.

\section{Statistical analysis}

Data analysis and graphs were completed using the R software program (R Development Core Team, 2006; R Foundation for Statistical Computing, Vienna, Austria). Spearman's $\rho$ and univariate regression were used to calculate the correlation of each of these identified DVH parameters and individual dependent binary variable (absence or presence of prolonged GT days, grade 3+ dysphagia, and severe weight loss). A logistic model, $\mathrm{p}=1$ / $\{1+\exp [-(\alpha+\beta *$ dose or volume of structure $)]\}$, was used to calculate the probability of developing prolonged GT days, grade $3+$ dysphagia, or severe weight loss. The unknown parameters $\alpha$ and $\beta$ were estimated with the maximum likelihood method. A test was also performed whether the hypothesis $\beta=0$ can be rejected. A p value of $<0.05$ was interpreted as being statistically significant from zero. Confidence intervals (95\%) were determined. Multivariate regression was not used due to the model instability caused by co-linearity between DVH parameters (V40, V50, V60, V65, Dmax, Dmean). Wilcoxon ranksum analysis was preformed to identify DVH parameters that statistically correlated with esophageal stricture. 


\section{Results}

\section{Swallowing outcomes after treatment}

At 3 months and 6 months after treatment, $87 \%$ and $44 \%$ of patients, respectively, were GT dependent (Table 2). The results of physician-assessed high grade dysphagia were consistent with that of GT dependence, given that majority of high grade dysphagia patients were grade 3 with GT dependence. Due to data redundancy, results of observer-assessed high grade dysphagia were not reported. Using the UWQOL instrument, 17 patients (44\%) reported high grade dysphagia at any point during or after treatment. The median percent of maximum weight loss was $12 \%$ (range, $-4 \%$ to $21 \%$ ).

More than half (54\%) of the patients had Grade 3 or 4 mucositis at some point after radiation, with $23 \%$ and 5\% having severe mucositis at 3-month and 6-month follow up evaluation respectively (Table 2). However, GT dependence did not improve as rapidly and still persisted in $87 \%$ and $44 \%$ of patients at 3 months and 6 months, respectively. There was a lack of temporal association between high grade mucositis and prolonged GT dependence of more than 192 days on statistical analysis ( $\mathrm{p}>$ $0.05)$. Grade 2 or higher xerostomia was found in $43 \%$ and $36 \%$ at 3-month and 6-month follow up evaluation respectively, and persisted in $31 \%$ at the last follow-up (Table 2).

Twelve of the patients with high grade dysphagia underwent TNE. Five of them developed stricture at the upper esophageal sphincter at the level of the cricopharyngeus muscle, including one with complete luminal stenosis. All of them underwent dilatation at the time of TNE to relieve any physical obstruction. Four out of the seven patients who underwent FEES had finding of moderate or severe aspiration to one of the diets. Eight patients had aspiration pneumonia work up, and only one of them was diagnosed.

The clinical factors listed in Table 1 were included in both univariate and multivariate analysis of prolonged GT dependence. Smoking (active smoking or smoking history

Table 2: Toxicity after treatment

\begin{tabular}{lcc}
\hline & 3 month (\%) & 6 month (\%) \\
\hline GT dependence & $87 \%$ & $44 \%$ \\
\hline $\begin{array}{l}\text { Self-reported dysphagia grade } \\
\text { Mucositis }\end{array}$ & $33 \%$ & $21 \%$ \\
\hline $\begin{array}{l}\text { grade 3 } \\
\text { Xerostomia grade 2 }\end{array}$ & $23 \%$ & $5 \%$ \\
\hline
\end{tabular}

Abbreviation: GT = gastrostomy tube within one year) was identified as the only significant factor predictive for prolonged GT dependence $(\mathrm{p}=0.03)$. Other clinical factors, including age, gender, history of alcohol use, KPS, tumor site, T stage, N stage, and type of chemotherapy regime are not associated with prolonged GT dependence. Similar analysis of high grade dysphagia revealed active smoking $(\mathrm{p}=0.03)$ and $\mathrm{T}$ stage $(\mathrm{p}=0.04)$ as significant factors. No other predisposing parameter was found to be statistically significant. In terms of severe weight loss, no predisposing parameter was identified to be statistically significant (data not shown). A total of 5 patients underwent post-treatment neck dissection. Both univariate and multivariate analysis did not reveal neck dissection as a significant factor for prolonged GT dependence, high grade dysphagia, or severe weight loss.

\section{DVH analysis for prolonged GT dependence}

The DVH parameters for all the swallowing structures (SMPC, IPC, CPI, and CE) were listed in Table 3. Significant factors $(\mathrm{p}<0.05)$ for prolonged GT dependence were revealed using Spearman's $\rho$ test and subsequent univariate logistic regression in an attempt to identify dose-volume effect for GT duration longer than 192 days versus less than 192 days. These factors are IPC V65 ( $p=0.003)$, IPC V60 ( $\mathrm{p}=0.002)$, IPC V50 ( $\mathrm{p}=0.042)$, IPC Dmean ( $=0.016)$, and CPI Dmax $(\mathrm{p}=0.011)$. CPI V60 has $\mathrm{p}$ value of 0.050. DVH analysis was also performed on a combined structure (IPC, CPI and CE). No statistically significant factor was identified ( $p>0.05)$.

The results of dose-response relationships and volumeresponse relationships for prolonged GT dependence are presented in Fig. 2. IPC V65 more than 30\%, IPC V60 more than 60\%, IPC Dmean more than $60 \mathrm{~Gy}$, and CPI Dmax more than 62 Gy predicted for a greater than $50 \%$ probability of developing prolonged GT dependence. For IPC V50, the dose/volume-response relationships results were not clinically meaningful.

In view of the strong dosimetric-clinical correlations for CPI and IPC, we repeated the above analysis with exclusion of three patients whose primary disease overlapped with the relevant structures (CPI and IPC). The same DVH parameters were observed as significant factors for prolonged GT dependence.

\section{DVH analysis for high grade dysphagia, severe weight loss, and stricture}

Spearman's $\rho$ test and subsequent univariate logistic regression analysis revealed significant associations between several dosimetric parameters and grade $3+$ patient-reported dysphagia. These factors are IPC V65 ( $\mathrm{p}=$ $0.040)$, CPI Dmax ( $p=0.037)$, and CPI V60 ( $p=0.046)$. Further analysis of dose-response relationships and volume-response relationships revealed that IPC V65 more 
Table 3: Swallowing structure DVH parameters (median value and range) and $p$ values for association with GT dependence

\begin{tabular}{|c|c|c|c|c|c|c|}
\hline & $\begin{array}{l}\text { V40 } \\
\text { (\%) }\end{array}$ & $\begin{array}{l}\text { V50 } \\
\text { (\%) }\end{array}$ & $\begin{array}{l}\text { V60 } \\
\text { (\%) }\end{array}$ & $\begin{array}{l}\text { V65 } \\
\text { (\%) }\end{array}$ & $\begin{array}{l}\text { Dmax } \\
\text { (Gy) }\end{array}$ & $\begin{array}{l}\text { Dmean } \\
\text { (Gy) }\end{array}$ \\
\hline \multicolumn{7}{|l|}{$C E$} \\
\hline$G T>192 d$ & $57(\mathrm{I}-86)$ & $24(0-79)$ & $0(0-33)$ & $0(0-1)$ & 61 (45-77) & $36(20-53)$ \\
\hline \multirow[t]{2}{*}{ GT $192 d$} & 65 (17-98) & $27(0-86)$ & $0(0-13)$ & $0(0-0)$ & 57 (44-76) & $36(2 \mid-56)$ \\
\hline & $p=2.671$ & $p=0.524$ & $p=0.173$ & $P=0.169$ & $p=0.238^{\prime}$ & $p=0.383$ \\
\hline \multicolumn{7}{|l|}{ CPI } \\
\hline$G T>192 d$ & $100(60-100)$ & $100(4-100)$ & $8(0-100)$ & $0(0-100)$ & $64(56-78)$ & $57(42-69)$ \\
\hline \multirow[t]{2}{*}{ GT $192 \mathrm{~d}$} & $100(93-100)$ & $95(3-100)$ & $0(0-73)$ & $0(0-27)$ & $58(53-67)$ & $53(43-62)$ \\
\hline & $p=0.512$ & $P=0.069$ & $P=0.050$ & $P=0.062$ & $P=0.011$ & $P=0.083$ \\
\hline \multicolumn{7}{|l|}{ IPC } \\
\hline$G T>192 d$ & $100(97-100)$ & $100(76-100)$ & $81(40-100)$ & $42(21-100)$ & 74 (66-79) & $64(54-70)$ \\
\hline \multirow[t]{2}{*}{ GT $192 \mathrm{~d}$} & $100(95-100)$ & $92(40-100)$ & $37(10-84)$ & $15(0-60)$ & 72 (68-77) & $55(46-68)$ \\
\hline & $p=0.367$ & $P=0.042$ & $p=0.002$ & $P=0.003$ & $P=0.057$ & $p=0.016$ \\
\hline \multicolumn{7}{|l|}{ SMPC } \\
\hline$G T>192 d$ & $100(60-100)$ & $100(56-100)$ & $94(45-100)$ & $72(21-93)$ & 76 (66-79) & $67(38-72)$ \\
\hline \multirow[t]{2}{*}{ GT $192 \mathrm{~d}$} & $100(84-100)$ & $97(70-100)$ & $90(25-100)$ & $60(0-95)$ & 76 (70-79) & $65(50-71)$ \\
\hline & $p=0.378$ & $P=0.072$ & $P=0.063$ & $P=0.091$ & $P=0.252$ & $P=0.086$ \\
\hline
\end{tabular}

Abbreviation: DVH = Dose-volume histogram; GT = gastrostomy tube; SMPC = superior and middle pharyngeal constrictor; IPC = inferior pharyngeal constrictors; $\mathrm{CPI}=$ cricoid pharyngeal inlet; and CE = cervical esophagus; $D$ max = maximum dose; $D$ mean $=$ mean dose.

than $65 \%$, CPI V60 more than 78\%, CPI Dmax more than 70 Gy were associated with more than $50 \%$ probability of developing high grade dysphagia. Similar analysis did not reveal statistically significant DVH predictors for severe weight loss (data not shown). Wilcoxon rank-sum analysis revealed significant associations between stricture and two dosimetric parameters (CPI V65, CPI Dmax).

\section{Discussion}

It has been a common observation that a correlation exists between dysphagia and radiation doses to the anatomic structures responsible for swallowing in patients undergoing definitive chemoradiation for head and neck cancer. However, the present study is the first to document a relationship between various dosimetric parameters and prolonged GT dependence. Notably, we were able to identify DVH parameters which were significantly associated with prolonged GT dependence, including V65 of the IPC, V60 of the IPC, mean dose to the IPC, and maximum dose to the CPI. Based on these dose/volume-response relationships, we currently recommend IPC V65 less than 15\%, IPC V60 less than 40\%, IPC Dmean less than 55 Gy, and CPI Dmax less than 60 Gy as potentially important DVH constraints to guide IMRT planning in an attempt to significantly reduce the risk of swallowing dysfunction and prolonged GT dependence.

Our findings demonstrate the importance of IPC and CPI dosimetric parameters for developing swallowing dysfunction and are consistent with those from several recently published studies. Caglar et al showed that a mean dose to the IPC of more than 54 Gy and IPC V50 of more than $50 \%$ were the most significant predictors for aspiration or stricture development [17]. Levendag et al identified dose-response relationship between dysphagia for solids $(\mathrm{p}<0.02)$ or aspiration episodes $(\mathrm{p}<0.02)$ and mean dose to IPC. A mean dose of 33 Gy to IPC was estimated as the threshold for $20 \%$ risk of dysphagia for solids [14]. Furthermore, Dornfeld et al reported that a more restrictive diet one year after treatment is significantly correlated with higher average dose delivered to the constrictor muscles (lateral pharygeal wall) at the level of false vocal cord [18]. Jensen et al demonstrated that dose above 60 Gy to the upper esophageal sphincter could result in higher risk of late swallowing dysfunction [19]. This well documented association between high dose to IPC or CPI and prolonged GT dependence was also supported by two earlier reports showing that patients were more likely to have prolonged GT dependence and high grade dysphagia when treated with extended-field IMRT rather than being treated with an upper IMRT fields junctioned with an anterior neck field. This is thought to be due to the presence of midline block in an anterior neck field to prevent unanticipated high dose radiation to structures including larynx, IPC, CPI and CE $[20,21]$.

The significant dose-volume effect relationships regarding prolonged GT dependence for IPC and CPI could be explained by the role of the upper esophageal sphincter (UES) in the normal swallowing process. The UES is a functional entity that is composed of three muscles: the IPC muscle, the CPI muscle, and the upper esophageal muscle. The UES opens by relaxation of the three closing muscles, traction by IPC and other muscles that attached to the hyoid bone and thyroid cartilage, anterior movement of the larynx, and pulsion of the bolus. The various 

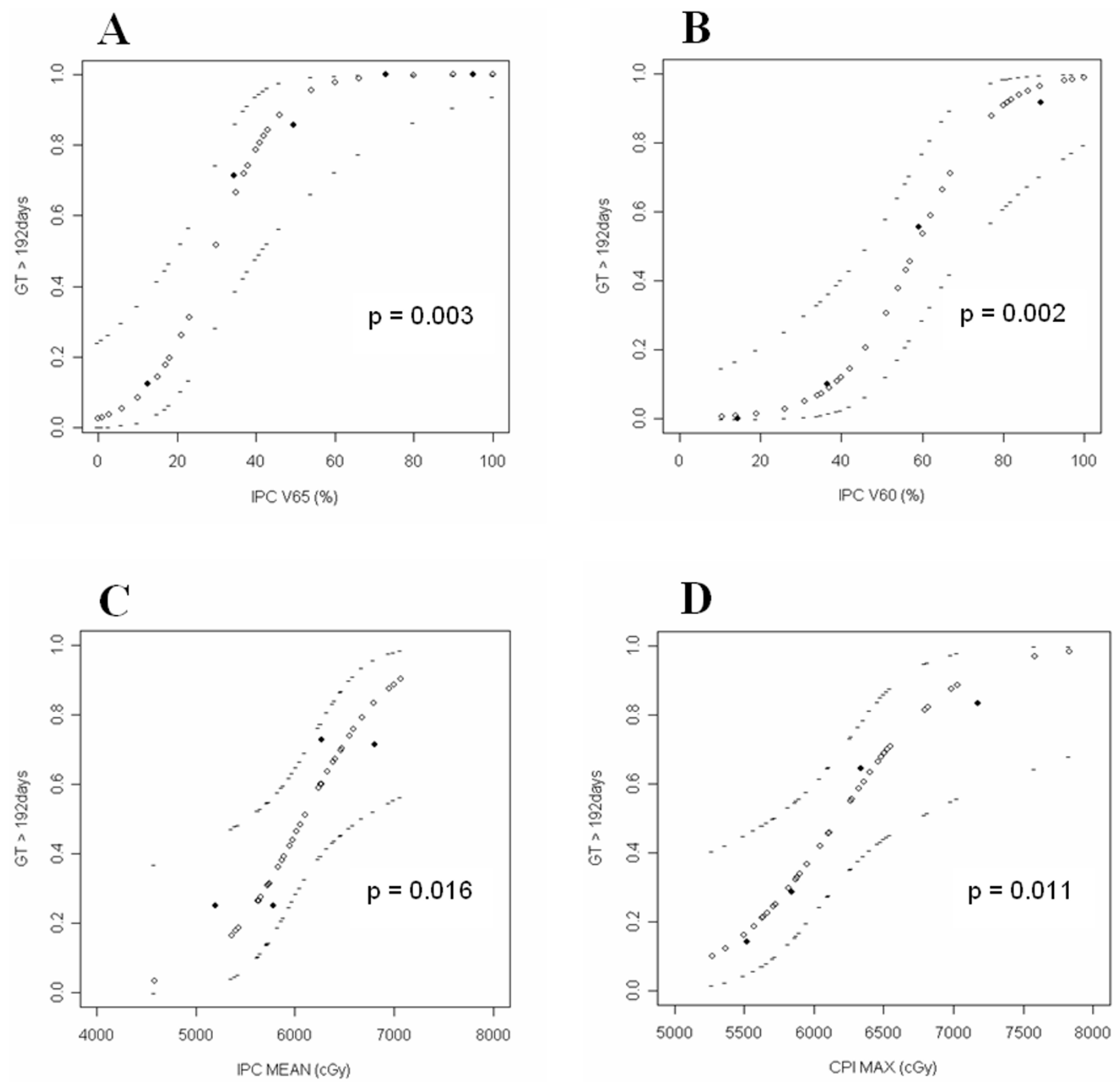

Figure 2

Volume-response or dose-response relationship for the average probability of having prolonged GT dependence and the volume of the IPC receiving more than $6500 \mathrm{cGy}(\mathrm{A})$, or $6000 \mathrm{cGy}(\mathrm{B})$, or the mean dose to the IPC (C), or maximum dose to CPI (D). GT = gastrostomy tube; IPC = inferior pharyngeal constrictors; CPI = cricoid pharyngeal inlet. The $\diamond$ lines plot the mean risk; the - lines plot the estimated upper and lower limits of $95 \%$ confidence interval. The points depict the observed values.

muscles of the UES behave differently during its many dynamic states, so that similar functions are accomplished by different muscles. Any impairment of the CPI and IPC could result in dysphagia. In addition, UES is considered a high pressure zone, with the highest pressure at the region around IPC where proprioceptive units were iden- tified. A cause of dysphagia could also be attributed in part to the failure of sensation and timely response to the bolus passing through this region. The importance of IPC and CPI is validated by our finding that patient-reported dysphagia was highly correlated with the dose to the two structures. 
In addition to the IPC and CPI, several other anatomic structures have been reported as dysphagia/aspiration related with significant dose-volume relationship. These structures include GSL and PC, with superior PC having the strongest dose-response association $[11,12]$. The importance of superior and middle PC for swallowing after radiation therapy was also shown by Teguh et al $[14,22,23]$. Although our study failed to find a significant a correlation between GT dependence and dose to the GSL, SMPC, or PC as a whole, this could potentially be explained by the differences in patient characteristics. In the above mentioned studies, only oropharynx and nasopharynx patients were included. As such, our results are consistent with those from Caglar et al that the mean dose or V50 to IPC, not the superior PC, were significant predictors for aspiration or stricture development [17].

Prolonged GT dependence is regarded by most head and neck cancer patients as contributing to compromised quality of life because it may cause infection and physical discomfort, distort patient's self-esteem, and induce anxiety, depression and social isolation [8]. This is of increasing concern in recent years when concurrent chemoradiation for tumor control and organ preservation has gained widespread practice but is associated with high rate of severe late dysphagia, including prolonged GT dependence [5]. Multiple large randomized trials testing intensified chemoradiation regimens reported GT rates of about $70 \%$, and chronic tube dependence of $10-20 \%$ $[3,6,7]$. In a recent study where $95 \%$ of the chemoradiation patients had prophylactic feeding tubes placed before treatment, Caglar et al reported prolonged GT dependence in $37 \%$ of the patients, with a median GT duration of 112 days after radiation completion [17]. Notably, we also identified smoking as a risk factor for GT dependence. The etiology for smoking induced dysphagia is likely multifactorial and related to prolonged tissue recovery secondary to nicotine induced hypoxia, the appetite reducing effects of nicotine, or mucosal irritation. Multiple previous works have similarly associated smoking with higher rates of toxicity including aspiration and esophageal stricture after radiation therapy $[24,25]$.

Notably, 5 out of the $12(42 \%)$ patients with high grade dysphagia developed upper esophageal stricture in this study. This high incidence could have resulted from detection bias, small patient number, or most likely, patient over-reliance on a GT which led to less swallowing and allowing scar and stricture formation. The last possibility is supported by results from Caudell et al who demonstrated a trend toward an association $(p=0.09)$ between GT dependence and pharyngeal stricture or stenosis [26]. Another explanation for this high incidence of stricture among patients with high grade dysphagia could be its relatation with CPI Dmax, which was significantly associated with both high grade dysphagia and stricture formation. In spite of this high incidence among patients with high grade dysphagia, the overall incidence of stricture in all patients is $12 \%(5 / 41)$ in our study, consistent with stricture rate of $17 \%-37 \%$ in other studies [17,26-28].

It is important to note that this study was retrospective with inherent limitations. First, the lack of systematic evaluation of some of the major end points of late dysphagia using TNE or FEES prevented more robust analysis using more objective endpoints. Another limitation of the study was the relatively short follow-up with a median duration of 15.6 months. However, most of the endpoint events occurred less than 1 year after treatment. Given the small number of events, we conjecture that a sub-analysis of swallowing function assessed at greater than 1 year after treatment would likely not change our findings. Nonetheless, we do acknowledge that further studies with more comprehensive objective endpoints with prolonged follow-up may be necessary to yield a more thorough evaluation. This is well exemplified in a recently published retrospective study of patients with more than 1 year follow-up, where a composite of 3 objective endpoints (GT dependence, aspiration, and pharyngoesophageal stricture) were successfully used as surrogates for severe longterm dysphagia [26].

It must also be recognized that the majority of patients in the present study presented with oropharynx cancer, and this fact may have biased our findings. Teguh et al, for instance, demonstrated that patients with base of tongue disease experienced more severe dysphagia than those with tumors at other sites [22]. In addition to oropharynx primary, the larynx, hypopharynx and pharyngeal wall were also found to predispose to dysphagia more so than other regions of the head and neck [26-29]. In contrast, however, Logemann et al showed no differences in the frequency of dysphagia across different head and neck disease sites [30], which is further supported by a large prospective study that excluded disease site as a statistically significant factor for quality of life changes among head and neck cancer treated with radiation therapy [31].

Lastly, we were unable to control for potentially confounding factors which may have also predisposed to swallowing dysfunction including severe mucositis, pretreatment dysphagia and post-treatment xerostomia. We acknowledge that the endpoints in this study, such as high grade dysphagia and prolonged GT dependence may have been confounded by the development of severe acute mucositis or its consequential late effects, such as submucosal edema, fibrosis, scarring, soft tissue necrosis, impaired sensory or motor function, and loss of mucosal compliance. This is based on the rationale that high dose radiation to a large volume of the constrictor muscles 
(resulting in high values of V60 and V65) also results in high dose to large volumes of mucosal surface which is believed to lead to more severe mucositis [32-34]. However, since mucositis tends to be self-limiting and an acute, rather than late side-effect of radiation therapy, we believe that the potential confounding effects are minimal. Furthermore, the present study demonstrated a lack of significant association between acute mucositis and prolonged GT dependence, which is consistent with the findings of a dissociation between acute mucositis and dysphagia by Mekhail et al [27] and it is supported by recent data from Anand et al showing no correlation between long-term dysphagia and acute mucositis (Grade $3,4)$ in spite of the severe mucosities that developed in $53 \%$ of locally advanced head neck cancer patients treated with IMRT [35]. In addition, we were unable to completely exclude the possibility of other confounding factors such as pre-treatment dysphagia or post-treatment severe xerostomia as confounding factors. Multiple studies suggested that very few patients with newly diagnosed head and neck cancer have severe dysphagia or aspiration prior to definitive treatment [29,36,37]. Moreover, we could not completely rule out xerostomia as a confounding factor in spite of the reduction in the risk of this symptom associated with parotid gland sparing IMRT. Xerostomia secondary to chemotherapy or radiation therapy has been suggested to significantly affect food bolus formation and swallowing function, and contribute significantly to dysphagia after chemoradiation $[38,39]$. In particular, Teguh et al demonstrated a strong correlation between dry mouth and sticky saliva with dysphagiarelated quality of life such as normalcy of diet and odynophagia [22]. As a result of these potential confounding factors, it remains difficult to definitively establish a cause-effect association in spite of the significant dose/volume-response relationship between some key DVH parameters and risk of prolonged GT dependence. A larger prospective study is required in the future to further investigate theses associations.

\section{Conclusion}

We provided evidence that prolonged GT is correlated with DVH parameters for patients with locally advanced head and neck cancer treated with definitive concurrent chemotherapy and IMRT. To minimize the risk of prolonged GT dependence, we currently strive to keep IPC V65 less than 15\%, IPC V60 less than 40\%, and maintain IPC Dmean less than 55 Gy, and CPI Dmax less than 60 Gy during IMRT planning in an attempt to decrease the risk of prolonged GT dependence. It should be noted that these guidelines are implemented on a case-by-case basis considering such factors as tumor extent and location. Future directions include large-scale prospective trials aiming to assess the clinical benefits gained by applying these dosimetric strategies. Lastly, the proposed dosimet- ric constraints should not replace the effort of early swallowing therapy and exercises which resulted in maximal swallowing recovery in several studies $[5,40]$.

\section{Competing interests}

The authors declare that they have no competing interests.

\section{Authors' contributions}

BL and AMC conceived of the study, and participated in its design, carried out data collection, data analysis, manuscript writing, and coordination. DL and DMR performed statistics analysis. DHL performed data collection regarding chemotherapy regimens and participated in manuscript writing. DGF and QL performed dysphagia data collection including TNE and FEES, and delineation of swallowing structures. KN and JC performed data collection regarding GT management. JAP performed physics consult on re-computation of DVH. All authors read and approved the final manuscript.

\section{Acknowledgements}

This study was presented in abstract form at the 2008 annual meeting of the American Society for Therapeutic Radiology and Oncology (ASTRO) in Boston, Massachusetts.

\section{References}

I. Adelstein DJ, Li Y, Adams GL, Wagner H Jr, Kish JA, Ensley JF, Schuller DE, Forastiere AA: An Intergroup phase III comparision of standard radiation therapy and two schedules of concurrent chemoradiotherapy in patients with unresectable squamous cell head and neck cancer. J Clin Oncol 2003, 21:92-98.

2. Denis F, Garaud P, Bardet E, Alfonsi M, Sire C, Germain T, Bergerot $P$, Rhein B, Tortochaux J, Calais G: Final results of the 94-0I French Head and Neck Oncology and Radiotherapy Group randomized trial comparing radiotherapy alone with concomitant radiochemotherapy in advanced-stage oropharynx carcinoma. I Clin Oncol 2004, 22:69-76.

3. Forastiere AA, Goepfert H, Maor M, Pajak TF, Weber R, Morrison W, Glisson B, Trotti A, Ridge JA, Chao C, Peters G, Lee DJ, Leaf A, Ensley J, Cooper J: Concurrent chemotherapy and radiotherapy for organ preservation in advanced laryngeal cancer. $N$ Engl J Med 2003, 349:209I-8.

4. Grégoire V, De Neve W, Eisbruch A, Lee N, Weyngaert D Van den, Van Gestel D: Intensity-modulated radiation therapy for head and neck carcinoma. Oncologist 2007, I 2:565-8.

5. Rosenthal DI, Lewin JS, Eisbruch A: Prevention and treatment of dysphagia and aspiration after chemoradiation for head and neck cancer. I Clin Oncol 2006, 24:2636-43.

6. Garden AS, Harris J, Vokes EE, Forastiere AA, Ridge JA, Jones C, Horwitz EM, Glisson BS, Nabell L, Cooper JS, Demas W, Gore E: Preliminary results of Radiation Therapy Oncology Group 9703: a randomized phase II trial of concurrent radiation and chemotherapy for advanced squamous cell carcinomas of the head and neck. J Clin Oncol 2004, 22:2856-64.

7. Adelstein DJ, Lavertu P, Saxton JP, Secic M, Wood BG, Wanamaker JR, Eliachar I, Strome M, Larto MA: Mature results of a phase III randomized trial comparing concurrent chemoradiotherapy with radiation therapy alone in patients with stage III and IV squamous cell carcinoma of the head and neck. Cancer 2000, 88:876-83.

8. Nguyen NP, Frank C, Moltz CC, Vos P, Smith HJ, Karlsson U, Dutta S, Midyett A, Barloon J, Sallah S: Impact of dysphagia on quality of life after treatment of head-and-neck cancer. Int J RadiatOncol Biol Phys 2005, 61:772-8.

9. Poulsen MG, Riddle B, Keller J, Porceddu SV, Tripcony L: Predictors of acute grade 4 swallowing toxicity in patients with stages 
III and IV squamous carcinoma of the head and neck treated with radiotherapy alone. Radiother Oncol 2008, 87:253-9.

10. Mendenhall WM, Morris CG, Amdur RJ, Hinerman RW, Mancuso AA Parameters that predict local control after definitive radiotherapy for squamous cell carcinoma of the head and neck. Head Neck 2003, 25:535-42.

II. Eisbruch A, Schwartz M, Rasch C, Vineberg K, Damen E, Van As C], Marsh R, Pameijer FA, Balm AJ: Dysphagia and aspiration after chemoradiotherapy for head-and-neck cancer: which anatomic structures are affected and can they be spared by IMRT? Int J Radiat Oncol Biol Phys 2004, 60: 1425-39.

12. Feng FY, Kim HM, Lyden TH, Haxer MJ, Feng M, Worden FP, Chepeha DB, Eisbruch A: Intensity-modulated radiotherapy of head and neck cancer aiming to reduce dysphagia: early doseeffect relationships for the swallowing structures. Int J Radiat Oncol Biol Phys 2007, 68: 1289-98.

13. Schmalfuss IM, Mancuso AA, Tart RP: Postcricoid region and cervical esophagus: normal appearance at $C T$ and $M R$ imaging. Radiology 2000, 2 1 4:237-46.

14. Levendag PC, Teguh DN, Voet P, Est $\mathrm{H}$ van der, Noever I, de Kruijf WJ, Kolkman-Deurloo IK, Prevost JB, Poll J, Schmitz PI, Heijmen BJ: Dysphagia disorders in patients with cancer of the oropharynx are significantly affected by the radiation therapy dose to the superior and middle constrictor muscle: a dose-effect relationship. Radiother Oncol 2007, 85:64-73.

15. Postma GN, Cohen JT, Belafsky PC, Halum SL, Gupta SK, Bach KK, Koufman JA: Transnasal esophagoscopy: revisited (over 700 consecutive cases). Laryngoscope 2005, II 5:32I-323.

16. Langmore SE, Schatz K, Olsen N: Fiberoptic endoscopic examination of swallowing safety: a new procedure. Dysphagia 1998, 2:216-9.

17. Caglar HB, Tishler RB, Othus M, Burke E, Li Y, Goguen L, Wirth LJ, Haddad RI, Norris CM, Court LE, Aninno DJ, Posner MR, Allen AM Dose to Larynx Predicts for Swallowing Complications After Intensity-Modulated Radiotherapy. Int J Radiat Oncol Biol Phys III8, 72:1 I 10-2008.

18. Dornfeld K, Simmons JR, Karnell L, Karnell M, Funk G, Yao M, Wacha J, Zimmerman B, Buatti JM: Radiation doses to structures within and adjacent to the larynx are correlated with long-term diet- and speech-related quality of life. Int J Radiat Oncol Biol Phys 2007, 68:750-7.

19. Jensen K, Lambertsen K, Grau C: Late swallowing dysfunction and dysphagia after radiotherapy for pharynx cancer: frequency, intensity and correlation with dose and volume parameters. Radiother Oncol 2007, 85:74-82.

20. Fua TF, Corry J, Milner AD, Cramb J, Walsham SF, Peters LJ: Intensity-modulated radiotherapy for nasopharyngeal carcinoma: clinical correlation of dose to the pharyngo-esophageal axis and dysphagia. Int J Radiat OncolBiol Phys 2007, 67:976-8I.

21. Lee NY, O'Meara W, Chan K, Della-Bianca C, Mechalakos JG, Zhung J, Wolden SL, Narayana A, Kraus D, Shah JP, Pfister DG: Concurrent chemotherapy and intensity-modulated radiotherapy for locoregionally advanced laryngeal and hypopharyngeal cancers. Int J RadiatOncol Biol Phys 2007, 69:459-68.

22. Teguh DN, Levendag PC, Noever I, van Rooij $P$, Voet $P$, Est $H$ van der, Sipkema D, Sewnaik A, Baatenburg de Jong RJ, de la Bije D, Schmitz PI: Treatment Techniques and Site Considerations Regarding Dysphagia-Related Quality of Life in Cancer of the Oropharynx and Nasopharynx. Int J Radiat Oncol Biol Phys 2008, 72:1119-27.

23. Teguh DN, Levendag PC, Sewnaik A, Hakkesteegt MM, Noever I, Voet $P$, Est $H$ van der, Sipkema D, van Rooij P, Baatenburg de Jong RJ, Schmitz PI: Results of fibroptic endoscopic evaluation of swallowing vs. radiation dose in the swallowing muscles after radiotherapy of cancer in the oropharynx. Radiother Oncol 2008, 89:57-63.

24. Jensen $K$, Jensen $A B$, Grau $C$ : Smoking has a negative impact upon health related quality of life after treatment for head and neck cancer. Oral Oncol 2007, 43:187-92.

25. Mangar S, Slevin N, Mais K, Sykes A: Evaluating predictive factors for determining enteral nutrition in patients receiving radical radiotherapy for head and neck cancer: $A$ retrospective review. Radiother Oncol 2006, 78:152-I58.

26. Caudell J, Shaner P, Meredith R, Bonner J, Locher JL, Nabell LM, Carroll WR, Magnuson JS, Spencer SA, Bonner JA: Factors associated with long-term dysphagia after definitive radiotherapy for locally advanced head-and-neck cnacer. Int J Radiat Oncol Biol Phys, Int J Radiat Oncol Biol Phys 2009, 73:41 0-5.

27. Mekhail TM, Adelstein DJ, Rybicki LA, Larto MA, Saxton JP, Lavertu $P$ : Enteral nutrition during the treatment of head and neck carcinoma: is a percutaneous endoscopic gastrostomy tube preferable to a nasogastric tube? Cancer 200I, 9I:I785-90.

28. Lee WT, Akst LM, Adelstein DJ, Saxton JP, Wood BG, Strome M, Butler RS, Esclamado RM: Risk factors for hypopharyngeal/upper esophageal stricture formation after concurrent chemoradiation. Head Neck 2006, 28:808-12

29. Machtay M, Moughan J, Trotti A, Gargen AS, Weber RS, Cooper JS, Forastiere A, Ang KK: Factors associated with severe late toxicity after concurrent chemoradiation for locally advanced head and neck cancer: an RTOG analysis. J Clin Oncol 2008, 26:358I-88.

30. Logeman JA, Rademaker AW, Pauloski BR, Lazarus CL, Mittal BB, Brockstein B, MacCracken E, Haraf DJ, Vokes EE, Newman LA, Liu D: Site of disease and treatment protocol as correlated of swallowing function in patients with head and neck cancer treated with chemoradiaiton. Head Neck 2006, 28:64-73.

3I. Langendijk JA, Doornaert P, Verdonck-de Leeuw IM, Leemans CR, Aaronson NK, Slotman BJ: Impact of late treatment-related toxicity on quality of life among patients with head and neck cancer treated with radiotherapy. J Clin Oncol 3776 , 26:3770-2008.

32. Rosenthal DI, Trotti A: Strategies for managing radiationinduced mucositis in head and neck cancer. Semin Radiat Oncol 2009, 19:29-34.

33. Dorr $\mathrm{W}$, Hendry JH: Consequential late effects in normal tissues. Radiother Oncol 200I, 6I:223-23I.

34. Trotti A, Bellm LA, Esptein JB, Frame D, Fuchs HJ, Gwede CK, Komaroff E, Nalysnyk L, Zilberberg MD: Mucositis incidence, severity and associated outcomes in patients with head and neck cancer receiving radiotherapy with or without chemotherapy: a systemic literature review. Radiother Oncol 2003, 66:253-262.

35. Anand A, Chaudhoory A, Shukla A, Negi PS, Sinha SN, Babu AA Munjal RK, Dewan AK, Kumar K, Doval DC, Vaid AK: Favourable impact of intensity-modulated radiation therapy on chronic dysphagia in patients with head and neck cnacer. $\mathrm{Br} J$ Radiol 2008, 81 I:865-7I.

36. Pauliski BR, Rademake AW, Logemann JA, Sten D, Beery Q, Newman L, Hanchett C, Tusant S, MacCracken E: Pretreatment swallowing function in patients with head and neck cancer. Head Neck 2000, 22:474-82.

37. Stenson KM, MacCracken E, List M, Haraf DJ, Brockstein B, Weichselbaum $R$, Vokes EE: Swallowing function in patients with head and neck cancer prior to treatment. Arch Otolaryngol Head Neck Surg 2000, 126:37|-7.

38. Logemann JA, Pauloski BR, Rademaker AW, Lazarus CL, Mittal B, Gaziano J, Stachowiak L, MacCracken E, Newman LA: Xerostomia: 12-month changes in saliva production and its relationship to perception and performance of swallow function, oral intake, and diet after chemoradiation. Head Neck 2003 . 25:432-7.

39. Hamlet S, Faull J, Klein B, Aref A, Fontanesi J, Stachler R, Shamsa F, Jones L, Simpson M: Mastification and swallowing in patients with postirradiation xerostomia. Int J Radiat Oncol Biol Phys I997, 37:789-96.

40. Shaker R, Easterling C, Kern M, Nitschke T, Massey B, Daniels S, Grande B, Kazandjian M, Dikeman K: Rehabilitation of swallowing by exercise in tube-fed patients with pharyngeal dysphagia secondary to abnormal UES opening. Gastroenterology 2002, I22:|3|4-2|. 